\title{
Enhanced Functional Annotation of Protein Sequences via the Use of Structural Descriptors
}

\author{
J eannine A. Di Gennaro,* Naomi Siew, $\dagger^{1}{ }^{1}$ Brian T. Hoffman,* Li Zhang, $\dagger^{, 2}$ J effrey Skolnick, $\ddagger$ \\ Lorna I. Neilson,* and J acquelyn S. F etrow*,3
}

\begin{abstract}
*GeneF ormatics, Incorporated, 5830 Oberlin Drive, Suite 200, San Diego, California 92121; †Department of Molecular Biology, The Scripps Research Institute, 10550 North Torrey Pines Road, La J olla, California 92037; and łLaboratory of Computational Genomes, Danforth Plant Science Center, 893 N. Warson Road, Creve Coeur, Missouri 63141
\end{abstract}

Received J anuary 8, 2001, and in revised form J une 4, 2001

In order to circumvent limitations of sequence based methods in the process of making functional predictions for proteins, we have developed a methodology that uses a sequence-to-structure-to-function paradigm. First, an approximate three-dimensional structure is predicted. Then, a threedimensional descriptor of the functional site, termed a F uzzy Functional Form, or FFF, is used to screen the structure for the presence of the functional site of interest (Fetrow et al., 1998; Fetrow and Skolnick, 1998). Previously, a disulfide oxidoreductase FFF was developed and applied to predicted structures obtained from a small structural database. Here, using a substantially larger structural database, we expand the analysis of the disulfide oxidoreductase FFF to the B. subtilis genome. To ascertain the performance of the FFF, its results are compared to those obtained using both the sequence alignment method BLAST and three local sequence motif databases: PRINTS, Prosite, and Blocks. The FFF method is then compared in detail to Blocks and it is shown that the FFF is more flexible and sensitive in finding a specific function in a set of unknown proteins. In addition, the estimated false positive rate of function prediction is significantly lower using the FFF structural motif, rather than the standard sequence motif methods. We also present a second FFF and describe a specific example of the results of its whole-genome application to $D$. melanogaster using a newer threading algorithm. Our results from all of these studies indicate that the addition of three-dimensional structural information adds significant value in the

\footnotetext{
${ }^{1}$ Current address: Department of Chemistry, Ben-Gurion University of the Negev, Beer-Sheva, I srael.

${ }^{2}$ Current address: Ernest Gallo Clinic and Research Center, 5858 Horton St, Suite 200, Emeryville, CA 94608.

3 To whom correspondence and reprint requests should be addressed. Fax: 858-450-1138. E-mail: jacque@geneformatics.com.
}

prediction of biochemical function of genomic sequences. ๑2 2001 Academic Press

Key Words: Bacillus subti lis; bi oi nformatics; disulfide oxidoreductase; Drosophila melanogaster; F F F (fuzzy functional form); functional annotation; protein function prediction; protein tyrosine phosphatase; structural genomics.

\section{INTRODUCTION}

F unctional genomics is a field born of necessities. These necessities include both the ability to identify novel biological targets in an environment of highly competitive research and the ability to exploit rapidly evolving volumes of genetic and genomic data. As a matter of practice, the approaches to structural/ functional genome analysis have developed as an amalgamation of existing technologies. Pattern recognition algorithms, artificial intelligence methods, computational chemistry and biology techniques, and even linguistic analytical methods have all been pressed into service as approaches to the task of interpreting protein function (Andrade and Valencia, 1998; Attwood et al., 1998; Andrade et al., 1999; Eisenhaber and Bork, 1999; Rindflesch et al., 1999; Pesole et al., 2000; Thomas et al., 2000; Tomic et al., 2000).

The goal of many functional genomics approaches has been to determine as many protein functions as possible in order to identify novel pharmaceutical targets. In fact, many recent cases document the successful identification of sequence motifs common to a set of related proteins (Henikoff and Henikoff, 1994; Attwood et al., 1998; Sonnhammer et al ., 1998; Bateman et al., 1999; H ofmann et al., 1999; Attwood, 2000). However, with the wealth of interest in such pursuits it has become apparent that approaches in which the predictions distinguish themselves in some fashion may be of significantly greater value 
than other competing methods. Distinctive approaches that make accurate predictions of function for greater numbers of proteins, provide accurate annotations of more distantly related proteins, accommodate the multi-functional nature of proteins, or are tailored to annotating protein classes that have garnered little attention are more likely to accomplish the desirable task of identifying a novel function for a previously unannotated protein sequence.

In the practice of making functional predictions for proteins, methods typically follow one of two avenues. The first approach is to identify a suitably homologous, previously annotated protein on the basis of sequence (primary structure) and/or overall structural similarities (secondary or tertiary structure) and transfer that protein's annotation to the query protein. The second means of functional prediction aims to develop or access a database of functional motifs, or templates, derived on the basis of knowledge of known protein sequences or structures and their function. Levels of resolution for both approaches may include comparisons of sequence similarity or sequence-based motifs, analysis of gross structural features (i.e., secondary structure or protein fold similarities), or a detailed three-dimensional feature homology assessment (i.e., specific residues involved in protein function). As might be expected, the level of complexity and expense (computational, temporal, and/or fiscal) generally increases as the level of resolution increases. For example, methods of functional annotation based on sequence-based motifs of known function include Blocks, PRINTS, Pfam, and Prosite (Henikoff and Henikoff, 1994; Attwood et al., 1998; Sonnhammer et al., 1998; Bateman et al., 1999; Hofmann et al., 1999; Attwood, 2000). These methods endeavor to capitalize on the conservation of sequence elements among functionally similar proteins. Generally, sequence-based annotation methods require relatively little in the way of computational time, development of the "rules" for analysis (termed "motifs") is generally facile (and sometimes even automated), and several of the tools are publicly available.

While methods of annotation on the basis of sequence similarity (such as BLAST (Altschul et al., 1997; Altschul and Koonin, 1998; Zhang et al., 1998)) or sequence motifs (such as Blocks, PRINTS, Pfam, and Prosite) have proven successful, they are limited by implicit assumptions underlying their methodology. For instance, some sequence similarity annotation methods rely upon existing database annotations for transfer of functional data. However, the prevalence of such methods and their application to whole genomes has served to inject significant levels of noise into database annotations
(Bork and Koonin, 1998; Karp, 1998). Because the annotation for an entire family might actually be transferred on the basis of sequence similarity, inaccurate annotations may be propagated rapidly.

Recently, development of elaborate automated methods of sequence annotation using overall threedimensional structural comparison has enabled whole-genome functional analyses. For example, Sanchez and Sali (1998) demonstrated via comparative modeling of the Saccharomyces cerevisiae genome that knowing only the fold of a protein is often sufficient to predict its approximate biochemical function, even when sequence-based methods are not capable of an accurate annotation. Another suite of tools has demonstrated the capability of generating functional predictions for $8-17 \%$ of the protein sequences of complete genomes (Andrade et al., 1999). However, such methods are al so subject to the potential pitfalls of sequence similarity-driven methods of functional annotation. The crux of the sequence similarity issue is at what level and to what extent function may be deduced from structure; even at higher levels of resolution of structural considerations, ambiguities arise from oversimplification of the structure-function relationship. The fact that the number of known folds in protein structure databases is quite tenable from a computational analysis standpoint may seem to enhance the attractiveness of a gross structural feature homology approach. However, biological reality actually indicates just the opposite; the presumption that fold similarities al one are sufficient to identify functional similarity is discredited in numerous cases (Koppensteiner et al., 2000). For example, paralogs (functionally divergent proteins) may share the same fold (such as $\alpha / \beta$ barrels (Brenner et al ., 1997)), and orthologs may exhibit dissimilar fold structures (eukaryotic and prokaryotic serine proteases have differing folds but all function as serine proteases (Fischer et al., 1994)). Hence there is a need to consider a residue-level approach, while retaining three-dimensional information, as a strategy for maximizing the specificity of a structurally based identification of function.

To this end, the challenges of a manageable approach to devising specific three-dimensional templates of protein function have been addressed by several groups. Artymiuk et al. (1994) focused on retrieving structures matching patterns of residue side-chain orientations by use of a graph-theory representation of atoms in both the template and query protein. Wallace et al. (1997) and Fischer et al. (1994) implemented automated geometric hashing algorithms to derive functional logic from structures in the Brookhaven Protein Data Bank. In addition, Wallace et al. (1997) have created a database of enzyme active 
site templates called PROCAT (see http://www. biochem.ucl.ac.uk/bsm/PROCAT/PROCAT.html). These three-dimensional functional motif databases serve the purpose of in silico screening of either existing databases of solved protein structures or novel experimental data regarding three-dimensional structural features. While these approaches have enjoyed success in creating templates for such purposes, their use is curtailed by the rigors and costs associated with the acquisition of high quality protein structures beyond those in publicly available databases. Given the current difficulty and expense of generating high quality structural data for proteins of unknown function, the use of computational methods that predict threedimensional structural features of protein sequences remains a relatively rapid and increasingly effective means of circumventing such limitations. However, because they rely on detailed knowledge of atom positions, these methods are not directly applicable to the approximate structures generated by today's state-ofthe-art structure prediction tools.

The advances in technologies geared toward calculation of protein structures has been well documented via the CASP (Critical Assessment of Techniques for Protein Structure Prediction) series, in which methods are assessed on the basis of their structure predictions for a common set of proteins (Murzin, 1999). Methods of protein three-dimensional structure prediction generally fall into the categories of homology modeling (Skolnick et al., 1997; Karplus et al., 1998; Tramontano, 1998; Fischer, 1999; Kolinski et al., 1999), fold recognition (Rost, 1995; Flockner et al., 1997; J ones et al., 1999; Kelley et al., 2000; Rychlewski et al., 2000; Skolnick, 2001) (for a review of early fold recognition methods, see Friesner and Gunn (1996)), and ab initio prediction (Skolnick et al., 1997; Moult, 1999; Osguthorpe, 2000). Homology modeling and the related method of protein threading rely upon the knowledge of existing structures via the identification of regions in which a template and query protein are structurally similar. Following the identification of a suitable template candidate protein(s), the method aligns the target sequence to the parent structure. It is then another task to build those regions of the query backbone not similar to any parent structure. Fold recognition algorithms aim to exploit knowledge of the relatively small number of folds that proteins utilize. The challenge then is to extract rules for fold assignment even in cases in which there is no obvious sequence homology between structures with related folds. These methods assign a fold, or series of folds, to a target sequence on the basis of these devised conventions. Finally, while ab initio is defined as "from the beginning," and although such methods aim to operate with limited knowledge dis- tilled from existing structures, many utilize data from known structures to cal culate scoring functions and assess prediction accuracy.

Our own approach toward identifying function in sequences of unknown structure has been to couple the strength of a library of three-dimensional descriptor-based templates termed FFF (Fuzzy Functional Form (Fetrow et al., 1998; Fetrow and Skolnick, 1998)) with a structure prediction method. Originally we utilized a hybrid threading algorithm (J aroszewski et al., 1998) and have recently moved to the use of Prospector (Skolnick, 2001) (Protein Structure Predictor Employing Combined Threading to Optimize Results: see http://bioinformatics. danforthcenter.org/services/prospector_doc.html ). Prospector improves upon other structure generating methods in that it does not limit prediction considerations to strictly fold recognition or true threading processes. Since "pure" fold recognition methods generally perform best in cases in which significant homology exists between probe and template proteins (Bryant, 1996), and protein threading methods are best suited more distant homology relationships (Murzin, 1999), the Prospector method captures the strength of each approach. The advantage of the marriage of fold recognition and true threading has recently been demonstrated (see http://bi oi nformatics.danforthcenter.org/services/ prospector_doc.html).

The impetus for the combination of the FFF and threading technologies was to implement the optimally suited approach to screening sequences of unknown structure for function. Constructed on the basis of the identities and the geometries between members of a defined subset of active site residues common to functionally similar proteins, FFFs encapsulate the minimum required physical and chemical structural elements for a specific biochemical function. Thus, proteins that exhibit the same function can be automatically identified, even if they are widely divergent in sequence and have dissimilar folds. For example, eukaryotic and bacterial serine proteases have different folds but they all function as serine proteases and share a common active site (Fischer et al., 1994), and they have been successfully identified using the FFF method (Zhang et al., 1998).

Here we describe the value of the use of a threedimensional template for function in annotating function for protein sequences. We provide specific examples of the new applications of both previously described (disulfide oxidoreductase (F etrow et al., 1998)) and novel (protein tyrosine phosphatase) FFFs in whole genome screenings to identify novel functional annotations for sequences found therein. We also provide comparisons of these annotations 
and those obtained via sequence-based functional annotation methods.

\section{MATERIALS AND METHODS}

The disulfide oxidoreductase and protein tyrosine phosphatase FFF. As described in previous work (Fetrow and Skolnick, 1998), the FFF for the disulfide oxidoreductase function was constructed of three active site residues: two cysteines, which are two residues apart in the sequence, forming a CXXC motif, and a proline distant in sequence but close in three-dimensional space. The second cysteine can be replaced by a serine and the same geometric constraints for a functional template are used. We utilized physicochemical data from three entries in the PDB in constructing the disulfide oxidoreductase FFF : laaz (a bacteriophage T4 thioredoxin) (Eklund et al., 1992), lego (an Escherichia coli glutaredoxin) (Xia et al., 1992), and 1dsb (an E. coli disulfide bond A protein) (Martin et al., 1993). With only the data from these PDB entries, our resulting functional template was sensitive enough to discriminately identify all 8 disulfide oxidoreductases in a test database of 1500 nonredundant PDB structures.

We compared the disulfide oxidoreductase FFF to two Blocks, the thioredoxin and glutaredoxin Blocks. The disulfide oxidoreductase FFF is designed to identify both thioredoxins and glutaredoxins, as well as disulfide isomerases, as all of these perform a similar chemical reaction at their active site. Given that, by definition, an FFF serves as a template of the underlying chemical functionality of a protein, the use of a single FFF corresponding to separate thioredoxin and glutaredoxin Blocks is appropriate.

The protein tyrosine phosphatase (PTP) FFF was constructed in the same manner described for the disulfide oxidoreductase FFF utilizing the following amino acids: a Cys that initiates catalysis via nucleophilic attack; an Arg that stabilizes the reaction intermediate; and an Asp that acts as a general acid (Sarmiento et al., 1998). As with the disulfide oxidoreductase FFF, our use of 10 PDB structures in defining the FFF yielded a template sensitive enough to detect all 23 PTPs in our test database of 4042 nonredundant PDB structures (this database is an expanded version of the one utilized in validating our disulfide oxidoreductase FFF).

Threading algorithm. The disulfide oxidoreductase FFF was applied to the Bacillus subtilis genome (http://www.ncbi.nlm. nih.gov/cgi-bin/E ntrez/framik?db=Genome\&gi=27). First, a threading algorithm ( aroszewski et al., 1998) was applied to the B. subtilis genome, as described previously (F etrow and Skol nick, 1998). The algorithm evaluated the threading results using three scoring functions, sq (sequence to sequence alignment), br (sequence to structure alignment), and tt (structure to structure alignment) ( aroszewski et al., 1998). Threading was performed against an extended database of 1501 known nonredundant structures, 8 of which are known disulfide oxidoreductases: 1bed (Hu et al., 1997), lego (Xia et al., 1992), lerv (Weichsel et al., 1996), 1fvk chain A (Guddat et al., 1997), 1kte (Katti et al., 1995), 1thx (Saarinen et al., 1995), 1tof (Mittard et al., 1997), and 2trx chain A (Katti et al., 1990). Next, the disulfide oxidoreductase FFF was applied to the threading results. The genome sequences that aligned correctly to these 8 structures, in accordance with the FFF, were further analyzed as described under Results.

The PTP FFF was applied to the open reading frames encoded in the left arm of chromosome 2 in the D. melanogaster genome (2486 sequences). In this experiment a more recent threading algorithm, Prospector (Skolnick, 2001), was used. Prospector uses two sequence-to-sequence alignment and two sequence-to-structure alignment scoring functions. For each type of scoring function, a closely related alignment and a distantly related alignment are calculated. Thus, a total of four scoring functions are employed in a Prospector analysis: close or distant sequence profiles, followed by application of a scoring function based on either sequence alone or sequence plus secondary structure plus pair interaction profiles. The closely related alignment is defined by the query sequence and the template sequences possessing 35-90\% sequence identity (as calculated by BLAST). The distantly related alignments are defined by the query sequence and the template sequences possessing a BLAST $E$ value $\leq 10$. Threading via Prospector was performed against a nonredundant structure database of 3332 proteins assembled by clustering the sequences of the protein data bank into groups with $\geq 40 \%$ sequence identity and selecting a representative from each cluster to populate the threading database. A Z score is calculated for each Drosophila sequence aligned to a structure by comparing normalized raw scores from each scoring function for each sequence. The PTP FFF was applied to the results of these threading calculations.

Conservation profile. To check the conservation of active site residues in a given sequence, we calculated a conservation profile (Zhang et al., 1998). First, a multiple sequence alignment was generated for the query sequence using BLAST (http:// www.ncbi.nlm.nih.gov/cgi-bin/BLAST/nph-psi_blast, (Altschul et al., 1997)), with an E value cutoff of 0.01 . Next, gaps were ignored, and for each column in the multiple sequence alignment the number of appearances of each residue was tallied. The resulting conservation profile depicts the degree of conservation among the set of related sequences in the multiple sequence alignment.

Searching for one sequence motif of the Blocks database against a whole genome. In order to find all the sequences in each genome that Blocks (http://blocks.fhcrc.org/blocks/blocks search.html, Blocks Database Version 11.0, J uly 1998 (Henikoff and Henikoff, 1994)) identifies as a thioredoxin, a screening of the B. subtilis was performed. One sequence motif ("block") in the Blocks database correlated with thioredoxin activity (Block BL 00194). It contains the active site cysteines motif, CXXC, and does not have a second sequence motif corresponding to the proline.

The B. subtilis genome was downloaded from the the NCBI web site (http://www.ncbi.nlm.nih.gov/cgi-bin/Entrez/framik? $\mathrm{db}=\mathrm{Genome} \& \mathrm{gi}=27)$. A search for the query Block was conducted on all sequences of the genome. Blocks aligns the query block to each sequence in the genome and looks for matching alignments. The result of each screening was a list of sequences, ranked by the Blocks score, that was found by Blocks to exhibit the thioredoxin motif.

Comparison of the FFF results with sequence motif databases. The threading/FFF results were compared to three known sequence motif databases: PRINTS (http://www.biochem.ud.ac.uk/ bsm/dbbrowser/PRINTS/PRINTS.html (Attwood et al., 1998), PRINTS20.0, released October 25, 1998); Prosite (http://www. expasy.ch/tools/scnpsit1.html (Hofmann et al., 1999), release 15, J uly 1998); and Blocks. These databases receive a sequence as input and output a list of sequence motifs, ranked by score, that may match the function of the query sequence. The thioredoxin and glutaredoxin families represent the disulfide oxidoreductase function in these local sequence motif databases. PRINTS uses a three-element fingerprint for thioredoxin (PS00160) and glutaredoxin (PS00421): one for the active site cysteines motif, CXXC; one for the proline motif; and a third one. Prosite uses only one signature containing the active site cysteines for thioredoxin (pattern PS00194) and glutaredoxin (pattern PS00195). Blocks uses one block for thioredoxin, BL00194, containing the active site cysteines motif, CXXC, and two blocks for glutaredoxin: BL00195A, encompassing the active site cysteines motif, and $B L 00195 B$, including the proline. Each of the $B$. subtilis protein sequences found by the FFF to contain the disulfide oxidoreduc- 
TABLE I

Proteins in the B. subtilus Genome Predicted to Have the Thiol-Disulfide Oxidoreductase Active Site

\begin{tabular}{|c|c|c|c|c|c|c|c|c|c|}
\hline \multirow[b]{2}{*}{ Sequence } & \multirow[b]{2}{*}{ Thrd/FFF } & \multirow[b]{2}{*}{$\mathrm{CP}$} & \multicolumn{3}{|c|}{ Functional motif } & \multicolumn{3}{|c|}{ Active site } & \multirow[b]{2}{*}{ Database description } \\
\hline & & & $\mathrm{Pr}$ & PS & $\overline{\mathrm{BI}}$ & $\mathrm{C}$ & $\mathrm{C} / \mathrm{S}$ & $P$ & \\
\hline $\operatorname{tr} x A$ & $x$ & $x$ & $x$ & $\times 2$ & $x$ & 29 & 32 & 73 & Thioredoxin \\
\hline yusE & $x$ & $x$ & $\times 2$ & $\times 3$ & $x$ & 26 & 29 & 69 & Possible thioredoxin \\
\hline ykvV & $x$ & $x$ & $x^{b}$ & $X 4$ & $x$ & 65 & 68 & 135 & Possible thioredoxin \\
\hline resA & $\underline{x}$ & $\underline{x}$ & $\underline{x}$ & $\times 5$ & $\underline{x}$ & 76 & 79 & 143 & Possible cyt-c biogen protein \\
\hline ytpP & $\bar{x}$ & $\bar{x}$ & $\bar{x}$ & $\overline{-}$ & $\bar{x}$ & 28 & 31 & 71 & Possible thioredoxin \\
\hline yvgV & $\mathrm{X}$ & $x$ & $x$ & - & $x$ & 69 & 72 & 193 & Unknown \\
\hline yneN & $\mathrm{X}$ & $x$ & $x$ & - & $x$ & 71 & 74 & 139 & Possible dsb \\
\hline$y d b P$ & $x$ & $x$ & $x$ & - & $x$ & 29 & 32 & 72 & Possible thioredoxin \\
\hline yoll & $x$ & $x$ & $x$ & - & $\times 3$ & 58 & 61 & 104 & Possible thioredoxin \\
\hline ytnl & $x$ & $x$ & $x$ & - & $\times 3$ & 15 & 18 & 57 & Unknown \\
\hline yosR & $x$ & $x$ & $x$ & - & $\times 4$ & 11 & 14 & 49 & Possible thioredoxin \\
\hline$y d b E$ & $x$ & $x$ & - & - & $X$ & 41 & 44 & 110 & Unknown \\
\hline ykuV & $\underline{x}$ & $\underline{x}$ & $=$ & $=$ & $\underline{x}$ & 41 & 44 & 112 & Unknown \\
\hline yuzD & $\bar{x}$ & $\bar{x}$ & 二 & 二 & 二 & 16 & 19 & 76 & Unknown \\
\hline ydfQ & $\underline{x}$ & $\underline{x}$ & $=$ & $=$ & $=$ & 32 & 35 & 75 & Possible thioredoxin \\
\hline ywbo & $\bar{x}$ & $?$ & 二 & 二 & $\overline{X 4}$ & 13 & 16 & 171 & Unknown \\
\hline yqiw & $x$ & $?$ & - & - & - & 52 & 54 & 103 & Unknown \\
\hline yqkB & $x$ & $?$ & - & - & - & 34 & 36 & 63 & Unknown \\
\hline yczA & $x$ & $?$ & - & - & - & 12 & 15 & 25 & Unknown \\
\hline ytxJ & $x$ & $?$ & - & - & - & 31 & 34 & 79 & Unknown \\
\hline
\end{tabular}

a Abbreviations used: Thrd/FFF, top six alignments of sequences from the B. subtilis genomes to the sequences of 1 bed, lego, lerv, 1 fvk (chain A), 1kte, 1thx, 1tof or 2trx (chain A), using an older threading algorithm (J aroszewski et al., 1998), followed by analysis of the resulting sequence-sequence alignment for the active site residues specified by the FFF for the thiol-disulfide oxidoreductase activity of the glutaredoxin/thioredoxin family (Fetrow et al., 1998); CP, conservation profile of homologous sequences shows if conservation of all three active site residues is greater than $50 \%$. The $E$ value cutoff is 0.01 ; a question mark (?) means that too few homologous sequences were found, so calculation of a conservation profile is not possible; Functional motif, search of each sequence found by Thread/FFF protocols against the local signature databases PRINTS (Pr; http://www.biochem.ucl.ac.uk/cgi-bin/attwood/SearchPrintsF orm2.pl), Prosite (PS; http://expasy.hcuge.ch/sprot/scnpsit1.html), or Blocks (Bl; http://www.blocks.fhcrc.org/blocks_search.html). The number following the $\mathrm{X}$ indicates the rank of the sequence in the search.

${ }^{\mathrm{b}}$ Of the three glutaredoxin/thioredoxin motifs found in PRINTS, only the C-C motif and a third one were found.

tase active site was used as input in the web version of these three motif databases. We then determined whether the sequence motif database identified the query sequence as either a thioredoxin or glutaredoxin, and, if so, at what position the motif database ranked the disulfide oxidoreductase for this sequence.

\section{RESULTS}

Enhanced data mining using FFFs: Mining the B. subtilis genome with the disulfide oxidoreductase FFF. To evaluate the effectiveness of using FFFs for biochemical function annotation across whole sets of genome sequences, the disulfide oxidoreductase FFF was used to screen models created from a threading alignment. Table I shows all sequences in the $\mathrm{B}$. subtilis genome that were identified by the FFF as having the disulfide oxidoreductase structural motif. To validate these results, a conservation profile was calculated for each sequence (Zhang et al., 1998-see Methods) and the conservation of the identified active site residues in each sequence was determined (Table I, column labeled "CP"). Our emphasis in calculating the conservation profile was on the conservation of the three active site residues identified by the FFF - the two cysteines and the proline. In most cases, the active site residues of the sequences that were identified by threading/FFF as disulfide oxidoreductases were conserved. Overall, in eight genomes analyzed (data not shown; B. subtilis data in Table I shown as example), $72 \%$ of the sequences identified by this FFF as disulfide oxidoreductases had conserved active site residues, while $14 \%$ of the sequences did not have enough homologous sequences to calculate a conservation profile, and another $14 \%$ of the sequences did not have conserved active site residues. Thus, for nearly three quarters of the identified sequences, there was substantiating evidence that the prediction of the exact functional residues might indeed be correct.

We wanted, however, to delineate in more detail which of the FFF hits were true positives and which were false positives. One way to approach this question was to employ a number of function prediction tools and simply stipulate those sequences that were identified by a majority of methods as "true positives." The term "consensus positives" more accu- 
rately describes these sequences, as many lack experimental evidence regarding function, and ther efore their classification as true positives relies on the consensus between various methods. Thus, the sequences identified as disulfide oxidoreductases by subsequently applying the FFF to the threading models were analyzed using the local sequence signature databases PRINTS (Attwood et al., 1998), Prosite (Hofmann et al., 1999), and Blocks (Henikoff and Henikoff, 1994). To obtain the data in Table I, each motif database received a protein sequence as input and, after comparing this sequence to all the motifs in the database, each annotation tool provided a list of the top scoring motifs for that sequence. A BLAST analysis was also used to classify sequences as consensus positives, though these data are not shown in Table I.

In this study, we identified 13 consensus positive sequences in the $B$. subtilis genome. Of these 13 sequences, 1 is known to be a thioredoxin $(\operatorname{tr} x A)$. Of the remaining 12 sequences, 8 are annotated in the genome database as having disulfide oxidoreductase function, i.e., possible thioredoxin, possible cytochrome c biogenesis protein, or possible disulfide bond formation protein. All 13 consensus positive sequences are identified by the threading/FFF method.

The FFF identified two additional sequences (yuzD and ydfQ) as disulfide oxidoreductases. Neither of these sequences is identified by any publicly available tool, but the conservation profile analyses of these sequences supported the FFF function prediction. While these sequences were not identified by any of the sequence-based methods, and therefore may not be considered as consensus positives, their conservation profiles suggest that they may in fact be novel hits. Additionally, ydfQ has been shown to have properties similar to those of thioredoxin (Beloin et al., 1997); thus a literature report suggests that this function prediction made only by the FFF and not by other methods is indeed correct.

There were four sequences (yqiW, yqkB, yczA, $y$ txJ ) that did not have corroborating conservation profiles or supporting annotations from other annotation methods. In the same vein, ywbO does not fall in the consensus positive database and there is no supporting experimental evidence; therefore, it should be counted as a false positive in using the consensus method. In total, using a consensus model, the disulfide oxidoreductase FFF identified six false positives (yuzD, yqiW, yqkB, yczA, ytxJ , and $y w b O$ ) in the $\mathrm{B}$. subtilis genome. However, of these six proteins, our annotation of disulfide oxidoreductase is supported in the case of ywbo by Blocks analysis and in the case of yuzD by conservation profile analysis.

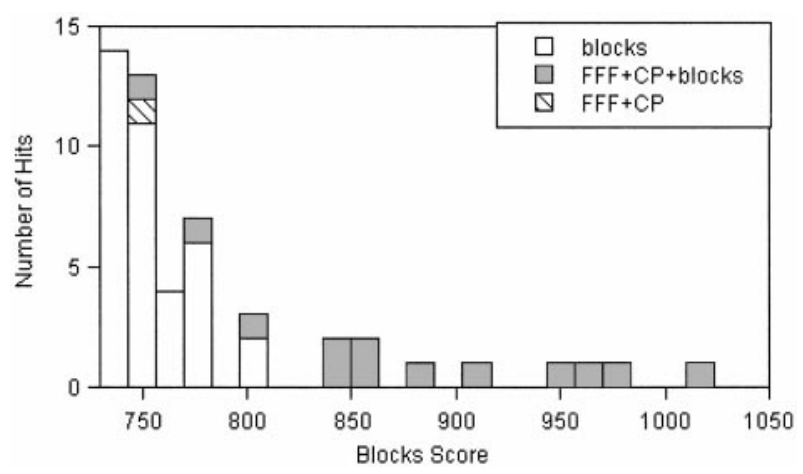

FIG. 1. The thioredoxin Blocks (Henikoff and Henikoff, 1994) score distributions for the $B$. subtilis genome with a comparison to the FFF results reported in Table I. The thioredoxin block (BL00194) was used to search each genome, as described under Materials and Methods. White bars are those sequences identified only by the Blocks sequence motif; gray bars are those sequences identified by the Blocks sequence motif, the FFF structural motif, and validated by the conservation profile (see Table I for a list of these sequences). In the analysis done here, these are counted as "true positives" or "consensus positives." The hashed bar represents one of the two sequences that were found by the FFF, validated by the conservation profile, and does not fall in the consensus positive group of sequences.

Whole genome mining: Structural motifs vs se quence motifs. In order to accurately assess the effectiveness of a specific example of an FFF, we have undertaken studies to generate comparisons of FFF results and those of other functional annotation tools. We estimated the ability of the FFF to distinguish between true and false positives via a direct comparison with Blocks (Henikoff and Henikoff, 1994), which, according to the results presented in Table I and other unpublished results, provides more predictions than either Prosite or PRINTS. In making this direct comparison to the FFF, the sequences from the $B$. subtilis genome were screened using one Blocks motif at a time. Our analyses employed three Blocks motifs: the thioredoxin block (BL 00194), which includes the active site cysteines, and the two glutaredoxin blocks (BL00195A and BL00195B), which encompass the cysteine and the proline motifs, respectively. As described under Materials and Methods, our use of a single FFF corresponding to both thioredoxin and glutaredoxin Blocks is appropriate given that the disulfide oxidoreductase encompasses both of these related biochemical functions. It should be emphasized that in this analysis each Block was screened against all sequences in the genome. Thus, three screenings were performed (one for each Block motif: BL00194, BL00195A, and BL 00195B), producing three lists of sequences ranked by their calculated significance score against each Block. The distribution of the top scoring sequences for this genome is shown in Fig. 1, 
which depicts the number of sequences identified by Blocks alone; by conservation profile, FFF, and Blocks; and by FFF and conservation profile, but not Blocks. The Blocks predictions were compared to the FFF predictions and to the conservation profile re sults from Table I.

To summarize the thioredoxin block search results, there was a general overlap of the FFF and Blocks results for the very high scoring sequences, but the results diverged for sequences that received lower scores. A predominant problem in interpreting Blocks results is a lack of guidance for a specific cutoff value that distinguishes clearly between true and false positives. For example, in the thioredoxin Blocks search there was neither a clustering of scores nor some threshold scoring gap clearly distinguishing a true ("consensus") positive from a false positive among sequences in this genome (scores ranging from 1021 to 735 ). The FFF identified sequences that received Blocks scores of 1021 to 808 as well as two additional FFF hits that received Blocks scores of 780 and 749 . B oth of the latter sequences have been previously annotated as possible thioredoxins, and BLAST predicts both of them to be thioredoxins. However, they fell within a Blocks score range with many other sequences of alternate functional annotations. Blocks identifies 7 additional sequences at scores of $770-780$ and 12 additional sequences at scores of 744-750 that the FFF did not identify, all of which are of unknown function or are known false positives.

As seen in Fig. 1, the analysis of thioredoxin Blocks figure showed that 25 false positives were found before all consensus positives were found. Since higher scores listed on the $X$ axis indicate higher confidence in the Blocks prediction, in order to identify all of the consensus hits (denoted FFF + $\mathrm{CP}+$ Blocks in Fig. 1), a consideration of sequences scoring 750 and above would be required. However, 25 sequences that were not consensus hits were also identified in this scoring range-in these analyses, these are considered the false positives. Similar FFF analyses shown in Table I yielded only 6 false positives, a significant reduction in false positive data for this genome.

FFFs extend structural information derived from threading al gorithms. An important issue in structural genomics is whether threading scores alone are sufficient to accurately predict the function of a sequence. To address this issue, the threading results for the $B$. subtilis genome were evaluated by three scoring functions, abbreviated sq br, and tt. From each raw score, a significance score was calculated (J aroszewski et al., 1998). The significance score distribution for the whole genome was plotted
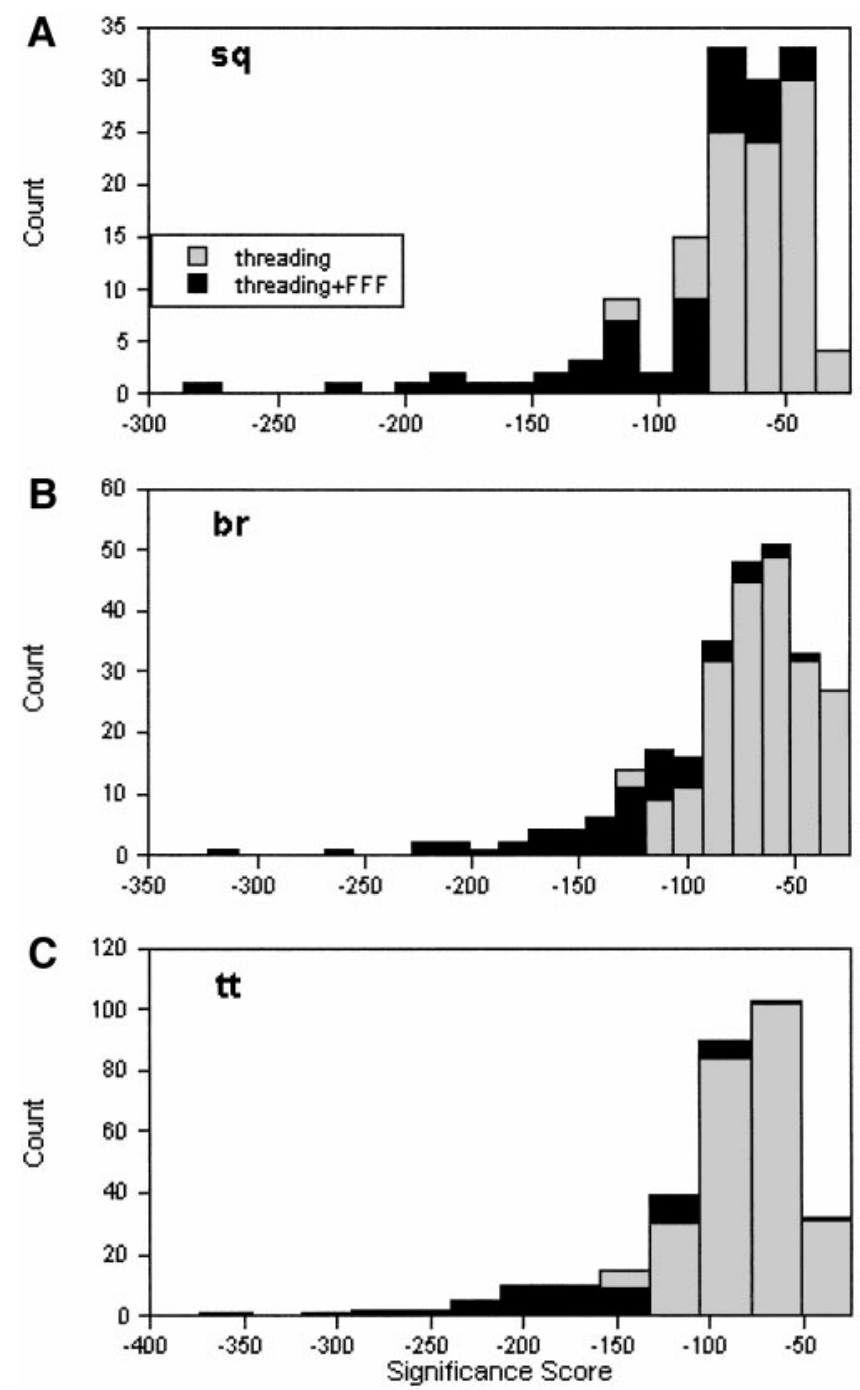

FIG. 2. The distribution of significance scores derived from the threading al gorithm applied to the B. subtilis genome. In each figure, the distribution of the three scoring functions, sq (sequence to sequence alignment), br (sequence to structure alignment), and tt (structure to structure alignment) are shown in A, $B$, and $C$, respectively. The more significant scores are more negative. The black bars represent sequences in the genome identified by the disulfide oxidoreductase FF F.

and alignments that were identified by the FFF as having the disulfide oxidoreductase active site were identified (Fig. 2). For each scoring function, there was a consensus of annotation between threading and the FFF analysis among sequences receiving a very significant score. But interpretation of the less significant scores indicates there is no obvious cutoff value that distinguishes between sequences identified by the FFF as potential disulfide oxidoreductases and those that are not. These results demonstrate that the raw threading score alone is not sufficient to predict the function of a given sequence. 
When the threading alignments and models are augmented by the application of a three-dimensional descriptor template (such as an FFF), sequences with a specific structure and function can be distinguished easily among all the sequences in a genome.

Structural descriptors enable accurate annotation of biological function in sequentially dissimilar proteins. Here we describe the development of an FFF for the function PTP. We have applied this FFF to structural predictions from Prospector, our latest threading algorithm (Skolnick, 2001). The PTP family of enzymes includes low-molecular-weight PTP, receptor-like PTP (RPTP), and dual-specificity TyrSer/Thr phosphatases. Members of this family cataIyze the removal of a phosphate moiety from tyrosine in phospoproteins. Given the low sequence and structural similarity among the diverse members of the protein tyrosine phosphatase family, development of an accurate set of three-dimensional descriptors of protein function would appear to be a daunting task. However, by identifying three amino acids essential for catalysis (a Cys that initiates catalysis via nucleophilic attack, an Arg that stabilizes the reaction intermediate, and an Asp that acts as a general acid (Sarmiento et al., 1998)) and their characteristic ranges in three-dimensional coordinates common to known PTPs, we successfully developed and validated a three-dimensional template of PTP function. The three key amino acids chosen as the basis of this template are structurally conserved within the PTP functional family, despite differing in their sequential positions (Fig. 3).

Figure 3 depicts the relative primary and tertiary structural positions of the PTP catalytic amino acids for the sequences of the human PTP 1B (PDB entry 2hnp (Barford et al., 1994)) and human low-molecular-weight PTP (PDB entry 5pnt (Zhang et al., 1998)). These two proteins exhibit only $2 \%$ sequence identity when aligned by clustalW (http://pbil. ibcp.fr/cgi-bin/npsa_automat.pl?page=/NPSA/npsa_ clustalw.html), yet perform the same biological function. In addition, the sequence position of the amino acids important for the phosphatase function differs between the two proteins (Fig. 3A). The Cys residue is located 6 amino acids from the Arg in both PTP sequences. In the sequence of $2 \mathrm{hnp}$, the Cys and Arg residues are located in the C-terminal end of the protein, but are alternatively located in the $\mathrm{N}$-terminal end of the PTP protein 5pnt. Likewise, these discrepancies in sequential positions are augmented by the fact that the position of the catalytic Asp is inverted between $2 \mathrm{hnp}$ and $5 \mathrm{pnt}$ with respect to the $\mathrm{C}$ - and N-termini of the protein (Fig. 3). Thus, the amino acids that make up the catalytic site fail to align in the primary sequence. The difference in the positions of Cys and Arg in the overall sequence of 2hnp and 5ptp would preclude use of the sequence of one to identify the chemical function of the other. While the potential exists for identifying the PTP function in both proteins using the CXXXXXR sequence motif common to both forms of PTP, doing so ignores the critical contribution of Asp to the chemistry of the catalytic mechanism.

A comparison of the overall structures of the two PTP proteins (Fig. 3B), and more specifically the active site (Fig. $3 \mathrm{C}$ ), reveals that the relative positions of these amino acids in three-dimensional space are conserved. In both 5 pnt and $2 \mathrm{hnp}$, the Cys is at the C-terminus of a $\beta$-strand, and Arg is at the $\mathrm{N}$-terminal end of an $\alpha$-helix. The Asp in 5pnt is at the C-terminal end of a $\beta$-strand and at the $\mathrm{N}$ terminal end of a $\beta$-strand in $2 \mathrm{hnp}$. Together, this conserved structure positions these key catalytic amino acids in a cleft on the surface of the proteins (Fig. 3B) and affords the basis for our structural motif that is designed to identify similarities in chemical function.

Mining the D. melanogaster genome using the protein tyrosine phosphatase FFF. In a whole genome comparative analysis of D. melanogaster, as deposited into the publidy available database GenBank (see http://www.ncbi.nlm.nih.gov/PMGifs/Genomes/ 7227.html), we have noted that the protein tyrosine phosphatase FFF has identified sequences for function that BLAST, Blocks, PRINTS, and Pfam have failed to identify. Here we list the results for one such sequence of unknown structure-a product of the chromosome referred to as $2 \mathrm{~L}$-identified by our PTP FFF as having protein tyrosine phosphatase activity. This sequence, listed in Fig. 4A, is 785 amino acids in length, and we hereafter refer to it by the Accession number gi| 7295991. The entry for gi| 7295991 in the Gene Ontology Consortium Gene Associations database (see http://www. geneontology.org/gene_association.fb) indicates that it has previously been associated with the cellular functions of "chaperone," as a component of the nucleus, and/or associated with the "DNA packaging" apparatus. These assessments were made on the basis of sequence similarities to other annotated proteins.

Results from threading via Prospector (Fig. 4B) indicate a Z score of 1.25 via the 3590 structural scoring method for threading of a fragment of gi| 7295991 to the PDB entry 1vhr (chain A), a score which is too insignificant to allow structure or function identification from threading al one. Subsequent analysis of the Prospector-predicted structure of sequence gi| 7295991 via our PTP FFF indicated that this protein possesses not only the requisite Cys, 
A

HUMAN PROTEIN TYROSINE PHOSPHATASE 1B (2hnp)

MEMEKEFEQIDKSGSWAAIYQDIRHEASDF PCRVAKLPKNKNRNRYRDVSPFDHSRIKLH QEDNDYINASLIKMEEAQRSYILTQGPLPNTCGHFWEMVWEQKSRGVVMLNRVMEKGSLK CAQYWPQKEEKEMIFEDTNLKLTLISEDIKSYYTVRQLELENLTTQETREILHFHYTTWP DFGVPESPASFLNFLFKVRESGSLSPEHGPVVVHCSAGIGRSGTFCLADTCLLLMDKRKD PSSVDIKKVLLEMRKFRMGLIQTADQLRFSYLAVIEGAKF IMGDSSVQDQWKELSHEDLE PPPEHIPPPPRPPKRILEPHN

HUMAN LOW MOLECULAR WEIGHT PROTEIN TYROSINE PHOSPHATASE (5pnt)

AEQATKSVLFVCLGNICRSPIAEAVFRKLVTDQNISENWRVDSAATSGYEIGNPPDYRGQ SCMKRHGI PMSHVARQITKEDFATFDYILCMDESNLRDLNRKSNQVKTCKAKIELLGSYD PQKQLIIEDPYYGNDSDFETVYQQCVRCCRAFLEKAH

B

Human Low Molecular Weight PTP

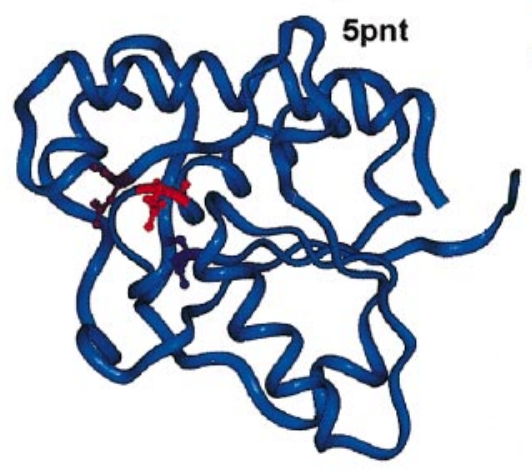

Q

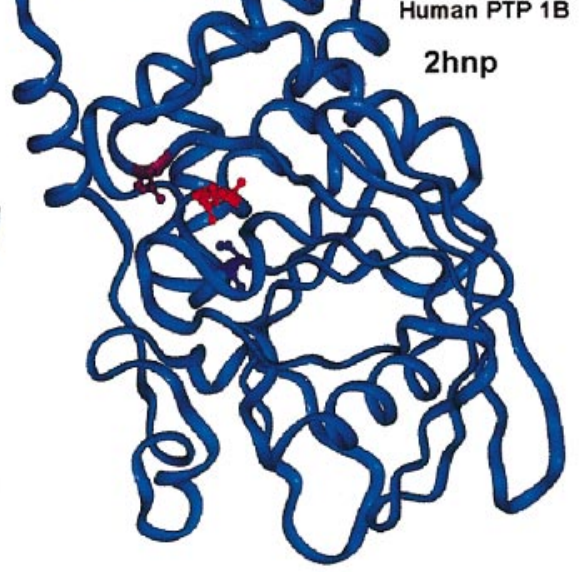

C

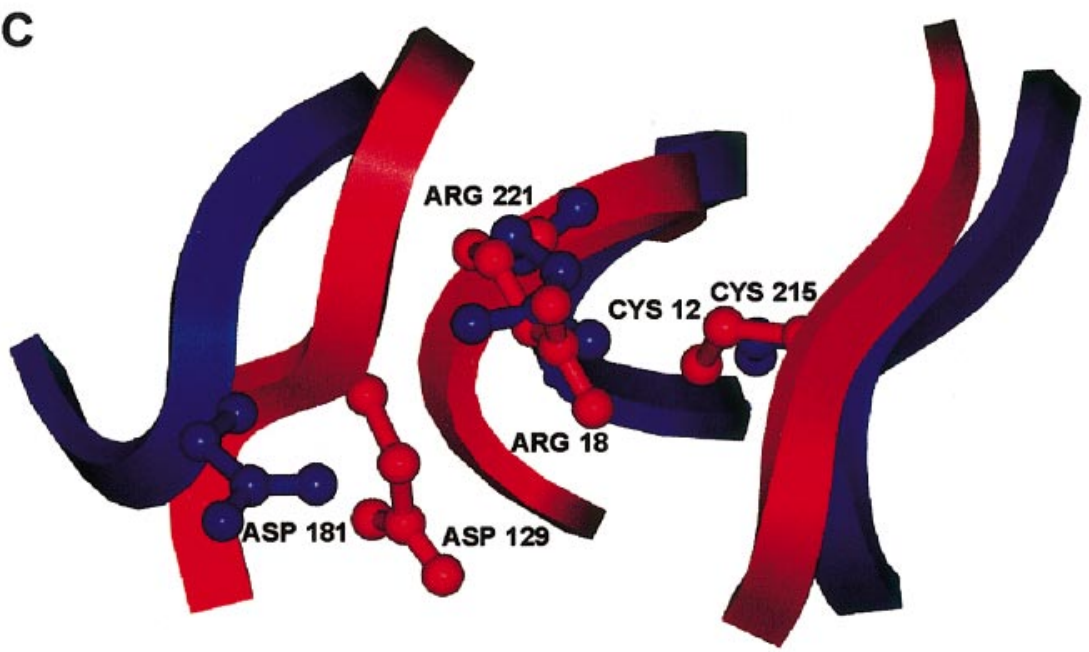

FIG. 3. Protein tyrosine phosphatase active site. (A) Sequences from the human protein tyrosine phosphatase 1B (PDB entry 2hnp) and human low-molecular-weight protein tyrosine phosphatase (PDB entry 5hnp). The Cys (blue), Arg (red), and Asp (purple) identified as important to the phosphatase activity are colored as indicated. (B) Structure of low-molecular-weight PTP and PTP 1B from human. The Cys, Arg, and Asp in both structures are colored as in A. (C) Magnified view of superimposed PTP active sites. The low-molecularweight PTP appears in red, and the PTP 1B appears in blue, with Cys, Arg and Asp depicted as ball and stick models. 


\section{A}

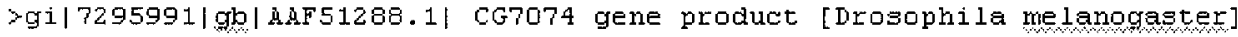
MSGNTHGLSUF P HF PDKFVSWGQE IHL YEVRRKD DHSOKSRL FY ISVNYL ANESR YQY ARCVA AS YHSDO P I I AVGL LDGKVG ICNF RD TYDSSUE YTP ROORMCTCL \& WNE LD AN I L A I GHDRHRND TCIT I UD IERGV PKE T ANF F GUGES ANS ICUD RNHRTV I AGMSQKM I KL F DL RQSNA TCOS IQTKTVQGL SVSPNGNYLCSY VDSVITL UD P RN I KSP L RQ IQSSKNHLQI AUCP TRT SLLSSLQRDSSY I TL YD I RSVD TDNSGE I YHVKR QISPF PARYQHSGKF S FVNCLSWHSRDFERALLL AD ALN ILDFRLFATLHTAHSNRRKLPLL MORFLYTP ASPTSTAI TP TQQQP TSSCSTNSGSSLDFSTPGGSFFNVD LL KPELFELDLVDETRORALED YG IKPDNK RF GELHL TP YLRNVUSTLNNVYSEDRLTGLKATLGINLGHTSEALMASSRIESOVLOTPEGINNSNKL IC YRSEQRD L ALQLCGWAFEQELDRF IDQL YANKE YSRAAM ICVF HLK IF HACN ILSS A ADNMRD FSMYR IT VIALSSFNADRCSSTWRNORSS ANMOI HDP HLRAVF SF L TNEKDNF D AVLKEEGUSLSDRMAF ACKYLSE TKL AD YU AQOIQA A I GGDLNGLLL TGESQDGID ILQS YND TSF DVQTVALVA INYF RQELF E DKR IQYU IASYL D HLNS WGL WEKRAELD IKIESIRFSSRSSRTVF LSCNFCGKSVSNALLDEPRPRSTTTSTNRLSS CPSCRKPLPRCSLCL

\section{B}

gi| 7295991
1vhri
gi| 7295991
lvhr
gil 7295991
lvhri
gi| 7295991
1vhri

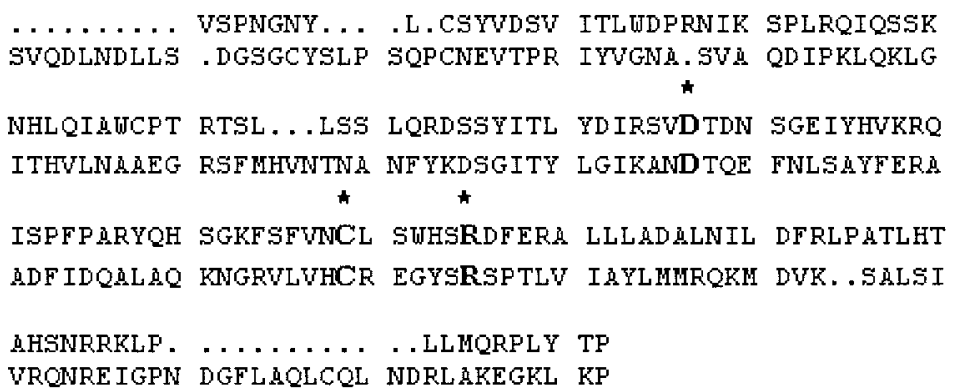

FIG. 4. (A) FASTA format and Accession information for the D. melanogaster sequence identified as a protein tyrosine phosphatase by the FFF template. (B) Resulting alignment from threading of fragments of the gi| 7295991 sequence to the PDB entry 1 vhr (chain A). Key catalytic residues are shown in bold type and marked by asterisks.

Arg, and Asp residues for PTP activity, but also that they are positioned appropriately in three-dimensional space to enable their roles in the PTP biochemical function. Although Prospector aligned gi| 7295991 to $1 \mathrm{vhr}$ with a low Z score (Z scores of $>4.5$ are typically considered biologically relevant), we again suggest that FFF s extend structural information derived from folding algorithms (Fig. 2). lvhr is annotated in the PDB as "Human-Vh1-Related Dual-Specificity Phosphatase" (Yuvaniyama et al., 1996). The function of dual specificity protein phosphatases (DSPs) has been reported to be the regulation of mitogenic signal transduction and the control of the cell cycle. Ivhr is less substrate-specific than PTPS, as it allows for the hydrolysis of phosphorylated serine, threonine, or tyrosine protein residues, whereas PTPs are restricted to only phosphotyrosine. However, given the variety of substrates acted upon by Ivhr, the Ivhr structure defines a conserved structural scaffold for both DSPs and PTPs that defines an overlap of biological function (Yuvaniyama et al., 1996). Hence, Prospector aligned gi| 7295991 to a protein with known PTP activity, and our PTP FFF subsequently identified gi| 7295991 as possessing the requisite three-dimensional structural motifs to infer that it is, in fact, a PTP.

We have also performed analyses by BLAST, Blocks, Pfam, and PRINTS (using default values) for gi| 7295991. None of these methods identified gi| 7295991 as a protein tyrosine phosphatase. Moreover, none of these methods identify gi| 7295991 as a phosphatase of any type. Even BLAST, which typically identifies protein sequences matching a particular function at some level (i.e., the $E$ value may be quite high for a particular BLAST function assignment), does not identify any similar sequences that are annotated as protein tyrosine phosphatases, up to the cutoff $\mathrm{E}$ value of 10 . The sequence-based tools associated the following functions most strongly with gi| 7295991: BLAST, "similar to yeast hypothetical protein ybk4" (E value = 1e-51); Blocks, "adhesin family signature" (combined E value = 0.093); Pfam, "Peptidase S15 X-Pro dipeptidyl-peptidase" (E value $=5.0$ ); PRINTS, "thyrotropin receptor precursor signature" $(E$ value $=58)$. These results strongly indicate that identification of gi| 7295991 as a protein tyrosine 
phosphatase is not readily accomplished utilizing existing annotation technologies. Given our confidence in the PTP FFF based upon our choice of structural motif components, and the fact that it identifies sequences in D. melanogaster as PTPs that sequence-based annotation methods fail to similarly annotate, we conclude that the FFF method of functional annotation holds a potential competitive advantage. Our method provides a novel hypothesis about biochemical function-one consistent with current annotations of cellular function via the Gene Ontology Consortium Gene Associations databasethat may now be experimentally validated.

\section{DISCUSSION}

While the use of overall three-dimensional structural information to extend functional annotation beyond the realm of sequence-based methods has been demonstrated to be effective (e.g., see Xu et al. (1999), the subsequent application of a protein function template clearly enhances the annotation process even further. Although results from threading provide insight into protein function on a fold-comparison basis, it is only with consideration of the geometries and constituents of the active sites of members of a related set of proteins that the most specific and robust predictions are possible. Using examples of two separate functions and proteins from two genomes, we have documented the abilities of this combination of structure and functional template and compared it with sequence-based analyses. Moreover, we have established novel functional predictions that provide hypotheses for future experimental exploration.

The $B$. subtilis genome was screened with the disulfide oxidoreductase FFF in search of sequences that exhibit this functionality. Sequences identified by the disulfide oxidoreductase FFF were also assessed for the conservation of their active site residues. In addition, these sequences were analyzed for function via the web versions of the three sequence motif databases, PRINTS, Prosite, and Blocks. N ot surprisingly, sequences identified by the FFF and annotated as disulfide oxidoreductases or as possible disulfide oxidoreductases had conserved active site residues and were also identified by at least two of the sequence motif databases.

In the comparison of the screenings by the FFF and by Blocks, a drawback to the Blocks method is the lack of a clear cutoff score that distinguishes between true and false positives. A possible reason for this difficulty in defining a clear scoring cutoff is that the Blocks motifs are specific to a certain family of proteins, i.e., thioredoxins or glutaredoxins, and therefore their sensitivity in identifying other disul- fide oxidoreductases is lower, thus producing scores that are not significant when compared to the many sequences found in a given genome. The advantage of the FFF is that it treats various protein families with equal sensitivity when searching for a specific function that is common to a few families and folds.

There are six sequences identified by the disulfide oxidoreductase FFF that do not fall in the consensus positive data set (Table I). Thus, at worst (since some evidence supports our annotation for two of these sequences), the FFF gives 6 false positives in screening the $\mathrm{B}$. subtilis genome. Our comparison of the thioredoxin Block and the disulfide oxidoreductase FFF demonstrates that Blocks identifies 25 false positives before finding all consensus positives. Therefore, the FFF gave a substantially lower false positive rate in whole genome screening, a competitive advantage when considering the time and expense of the biological verification of function for unknown sequences.

The noted discrepancy between the number of disulfide oxidoreductase sequences identified by the disulfide oxidoreductase FFF (6 potential false positives) and the thioredoxin Block (25 potential false positives) could be attributed to the fact that the thioredoxin Blocks includes only the active site cysteines CXXC motif. Based on these results, it might be expected that the glutaredoxin Block search would yield a better accord between Blocks and the FFF, since two Blocks are available: the cysteines block sequence motif (BL00195A) and the proline block (BL00195B). But our results indicated that there was still a large discrepancy in the performance of the glutaredoxin blocks and the FFF, even among the high scoring sequences (data not shown).

The use of threading scores without the subsequent application of a three-dimensional descriptor template is insufficient to optimally predict the function of a sequence. As the threading results for the B. subtilis genome indicate (Fig. 2), threading results, when considered alone, appear to fall prey to several sequence-based motif methods of functional annotation; interpretation of the less significant scores fails to delineate an obvious cutoff value that distinguishes between those sequences that are identified by the FFF as potential disulfide oxidoreductases and those that are not. These results demonstrate the added value of the use of structural descriptors for function when utilized in combination with threading results.

Using the particular case of the FFF for protein tyrosine phosphatase and our updated threading algorithm, we have demonstrated the circumvention of certain limitations inherent to sequence-based similarity or motif methods of functional annotation. Of the sequence-based methods, only BLAST annotated 
gi| 7295991 with a score of any confidence, and even in that instance, it was as having similarity to a yeast hypothetical protein. The failure of these sequencebased methods in identifying gi| 7295991 as a PTP is indicative of the exceptional strength of the FFF approach; while the sequence-based methods may be suitable at identifying PTPs that are similar to currently known PTP sequences, the PTP FFF is capable of identifying novel PTP sequences regardless of sequence similarity. Although the PTPs $2 \mathrm{hnp}$ and 5pnt are listed as members of separate superfamilies in the Structural Classification of Proteins database"(phosphotyrosine protein) phosphatases II" (http:// scop.mrc-Imb.cam.ac.uk/scop/data/scop.1.003.040. 001.002.001.html) and "phosphotyrosine protein phosphatases I" (http://scop.mrc-Imb.cam.ac.uk/scop/ data/scop.1.003.039.001.001.002.html), respectively, they are both accommodated by our PTP functional template. Since the FFF template is defined on the basis of the minimum required chemicostructural features common to all PTPs, any PTP, whether currently known or not, is likely to be accurately identified by the FFF.

The entry for gi| 7295991 in the Gene Ontology Consortium Gene Associations database indicates that it has previously been associated with the functions of "chaperone," as a component of the nucleus, and/or associated with "DNA packaging" apparatus. Certainly, these annotations are not contrary to our prediction of PTP activity for gi| 7295991. PTPs have been implicated in interactions with chaperone proteins (Fu and Galan, 1998; Montagna et al., 2000) and have been confirmed to be nuclear components (Misawa and Yamaguchi, 2000; Tsurusaki et al., 2000). Moreover, PTPs have been demonstrated to be participants in mitotic events, including an important role at the G1/S-phase transition (Nilsson and Hoffmann, 2000). Thus, our predicted biochemical function for gi| 7295991 is consistent with its previously annotated cellular function. The protein does appear to have a WD-repeat domain, but this is a separate domain from the putative phosphatase. In addition, WD repeats are found in proteins with a wide range of functions, including those involved in signal transduction, and specifically in subunits of phosphatases (Smith et al., 1999).

\section{CONCLUSION}

As with any alternative method, the use of FFFs in functional annotation is not failsafe. However, we have demonstrated that their use has proven to be highly successful in distinguishing between true ("consensus") and false positives when screening whole genomes. Combined with an accurate struc- tural prediction algorithm, such as Prospector, this method of the use of three-dimensional descriptors of function allows new insight into the subset of proteins for which sequence-based methods alone are not sufficient for functional annotation.

The authors acknowledge the editing expertise of Ruth Feldblum as instrumental in the preparation of the manuscript.

\section{REFERENCES}

Altschul, S. F., and Koonin, E. V. (1998) Iterated profile searches with PSI-BLAST-A tool for discovery in protein databases, Trends Biochem. Sci. 23, 444- 447.

Altschul, S. F., Madden, T. L., Schaffer, A. A., Zhang, J ., Zhang, Z., Miller, W., and Lipman, D. J . (1997) Gapped BLAST and PSI-BLAST: A new generation of protein database search programs, Nucleic Acids Res. 25, 3389-3402.

Andrade, M. A., Brown, N. P., Leroy, C., Hoersch, S., de Daruvar, A., Reich, C., Franchini, A., Tamames, J ., Valencia, A., Ouzounis, C., and Sander, C. (1999) Automated genome sequence analysis and annotation, Bioinformatics 15, 391- 412.

Andrade, M. A., and Valencia, A. (1998) Automatic extraction of keywords from scientific text: Application to the knowledge domain of protein families, Bioinformatics 14, 600-607.

Artymiuk, P. J ., Poirrette, A. R., Grindley, H. M., Rice, D. W., and Willett, P. (1994) A graph-theoretic approach to the identification of three-dimensional patterns of amino acid side-chains in protein structures, J. Mol. Biol. 243, 327-344.

Attwood, T. K. (2000) The quest to deduce protein function from sequence: The role of pattern databases, Int. J . Biochem. Cell Biol. 32, 139-155.

Attwood, T. K., Beck, M. E., Flower, D. R., Scordis, P., and Selley, J. N. (1998) The PRINTS protein fingerprint database in its fifth year, Nucleic Acids Res. 26, 304-308.

Barford, D., Flint, A. J ., and Tonks, N. K. (1994) Crystal structure of human protein tyrosine phosphatase 1B [see comments], Science 263, 1397-1404.

Bateman, A., Birney, E., Durbin, R., Eddy, S. R., Finn, R. D., and Sonnhammer, E. L. (1999) Pfam 3.1: 1313 multiple alignments and profile HMMs match the majority of proteins, Nucleic Acids Res. 27, 260-262.

Beloin, C., Ayora, S., Exley, R., Hirschbein, L., Ogasawara, N., Kasahara, Y., Alonso, J. C., and Hegarat, F. L. (1997) Characterization of an Irp-like (IrpC) gene from Bacillus subtilis, Mol. Gen. Genet. 256, 63-71.

Bork, P., and Koonin, E. V. (1998) Predicting functions from protein sequences-where are the bottlenecks? Nature Genet. 18, 313-318.

Brenner, S. E., Chothia, C., and Hubbard, T. J . (1997) Population statistics of protein structures: Lessons from structural classifications, Curr. Opin. Struct. Biol. 7, 369-376.

Bryant, S. H. (1996) Evaluation of threading specificity and accuracy, Proteins 26, 172-185.

Eisenhaber, F., and Bork, P. (1999) Evaluation of human-readable annotation in biomolecular sequence databases with biological rule libraries, Bioinformatics 15, 528-535.

Eklund, H., Ingelman, M., Soderberg, B. O., Uhlin, T., Nordlund, P., Nikkola, M., Sonnerstam, U., J oelson, T., and Petratos, K. (1992) Structure of oxidized bacteriophage T4 glutaredoxin (thioredoxin). Refinement of native and mutant proteins, J . Mol. Biol. 228, 596- 618.

Fetrow, J. S., Godzik, A., and Skolnick, J . (1998) Functional 
analysis of the Escherichia coli genome using the sequence-tostructure-to-function paradigm: Identification of proteins exhibiting the glutaredoxin/thioredoxin disulfide oxidoreductase activity, J. Mol. Biol. 282, 703-711.

Fetrow, J . S., and Skolnick, J . (1998) Method for prediction of protein function from sequence using the sequence-to-structure-to-function paradigm with application to glutaredoxins/ thioredoxins and T1 ribonucleases, J. Mol. Biol. 281, 949-968.

Fischer, D. (1999) Modeling three-dimensional protein structures for amino acid sequences of the CASP3 experiment using sequence-derived predictions, Proteins 37, 61- 65.

Fischer, D., Wolfson, H., Lin, S. L., and Nussinov, R. (1994) Three-dimensional, sequence order-independent structural comparison of a serine protease against the crystallographic database reveals active site similarities: Potential implications to evolution and to protein folding, Protein Sci. 3, 769-778.

Flockner, H., Domingues, F. S., and Sippl, M. J . (1997) Protein folds from pair interactions: A blind test in fold recognition, Proteins Suppl. 129-133.

Friesner, R. A., and Gunn, J . R. (1996) Computational studies of protein folding, Annu. Rev. Biophys. Biomol. Struct. 25, 315342.

Fu, Y., and Galan, J . E. (1998) I dentification of a specific chaperone for SptP, a substrate of the centisome 63 type III secretion system of Salmonella typhimurium, J . Bacteriol. 180, 33933399.

Guddat, L. W., Bardwell, J . C., Glockshuber, R., Huber-Wunderlich, M., Zander, T., and Martin, J . L. (1997) Structural analysis of three His32 mutants of DsbA: Support for an electrostatic role of His32 in DsbA stability, Protein Sci. 6, 1893-1900.

Henikoff, S., and Henikoff, J . G. (1994) Protein family classification based on searching a database of blocks, Genomics 19, 97-107.

Hofmann, K., Bucher, P., Falquet, L., and Bairoch, A. (1999) The PROSITE database, its status in 1999, Nucleic Acids Res. 27, 215-219.

Hu, S. H., Peek, J. A., Rattigan, E., Taylor, R. K., and Martin, J . L. (1997) Structure of TcpG, the DsbA protein folding catalyst from Vibrio cholerae, J. Mol. Biol. 268, 137-146.

J aroszewski, L., Rychlewski, L., Zhang, B., and Godzik, A. (1998) Fold prediction by a hierarchy of sequence, threading, and modeling methods, Protein Sci. 7, 1431-1440.

J ones, D. T., Tress, M., Bryson, K., and Hadley, C. (1999) Successful recognition of protein folds using threading methods biased by sequence similarity and predicted secondary structure, Proteins 37, 104-111.

Karp, P. D. (1998) What we do not know about sequence analysis and sequence databases [editorial], Bioinformatics 14, 753754.

Karplus, K., Barrett, C., and Hughey, R. (1998) Hidden Markov models for detecting remote protein homologies, Bioinformatics 14, 846- 856.

Katti, S. K., LeMaster, D. M., and Eklund, H. (1990) Crystal structure of thioredoxin from Escherichia coli at $1.68 \mathrm{~A}$ resolution, J. Mol. Biol. 212, 167-184.

Katti, S. K., Robbins, A. H., Yang, Y., and Wells, W. W. (1995) Crystal structure of thioltransferase at $2.2 \mathrm{~A}$ resolution, Protein Sci. 4, 1998-2005.

Kelley, L. A., MacCallum, R. M., and Sternberg, M. J . (2000) Enhanced genome annotation using structural profiles in the program 3D-PSSM, J. Mol. Biol. 299, 499-520.

Kolinski, A., Rotkiewicz, P., Ilkowski, B., and Skolnick, J . (1999) A method for the improvement of threading-based protein models, Proteins 37, 592- 610.
Koppensteiner, W. A., Lackner, P., Wiederstein, M., and Sippl, M. J . (2000) Characterization of novel proteins based on known protein structures, J . Mol. Biol. 296, 1139-1152.

Martin, J. L., Bardwell, J. C., and Kuriyan, J. (1993) Crystal structure of the DsbA protein required for disulphide bond formation in vivo, Nature 365, 464- 468.

Misawa, H., and Yamaguchi, M. (2000) Intracellular signaling factors-enhanced hepatic nuclear protein binding to TTGGC sequence in the rat regucalcin gene promoter: Involvement of protein phosphorylation [In Process Citation], Biochem. Biophys. Res. Commun. 279, 275-281.

Mittard, V., Blackledge, M. J ., Stein, M., J acquot, J . P., Marion, D., and Lancelin, J. M. (1997) NMR solution structure of an oxidised thioredoxin $\mathrm{h}$ from the eukaryotic green alga Chlamydomonas reinhardtii, Eur. J . Biochem. 243, 374-383.

Montagna, L. G., I vanov, M. I., and Bliska, J . B. (2000) Identification of residues in the amino-terminal domain of the Yersinia tyrosine phosphatase that are critical for substrate recognition, J . Biol. Chem.

Moult, J . (1999) Predicting protein three-dimensional structure, Curr. Opin. Biotechnol. 10, 583-588.

Murzin, A. G. (1999) Structure classification-based assessment of CASP3 predictions for the fold recognition targets, Proteins 37, 88-103.

Nilsson, I., and Hoffmann, I. (2000) Cell cycle regulation by the Cdc25 phosphatase family, Prog. Cell Cycle Res. 4, 107-114.

Osguthorpe, D. J . (2000) Ab initio protein folding, Curr. Opin. Struct. Biol. 10, 146-152.

Pesole, G., Liuni, S., and D'Souza, M. (2000) PatSearch: A pattern matcher software that finds functional elements in nucleotide and protein sequences and assesses their statistical significance, Bioinformatics 16, 439- 450.

Rindflesch, T. C., Hunter, L., and Aronson, A. R. (1999) Mining Molecular Binding Terminology from Biomedical Text, Proc. AMIA Symp., pp. 127-131.

Rost, B. (1995) TOPITS: Threading one-dimensional predictions into three-dimensional structures, Ismb 3, 314-321.

Rychlewski, L., J aroszewski, L., Li, W., and Godzik, A. (2000) Comparison of sequence profiles. Strategies for structural pre dictions using sequence information, Protein Sci. 9, 232-241.

Saarinen, M., Gleason, F. K., and Eklund, H. (1995) Crystal structure of thioredoxin-2 from Anabaena, Structure 3, 10971108.

Sanchez, R., and Sali, A. (1998) Large-scale protein structure modeling of the Saccharomyces cerevisiae genome, Proc. Natl. Acad. Sci. USA 95, 13597-13602.

Sarmiento, M., Zhao, Y., Gordon, S. J ., and Zhang, Z. Y. (1998) Molecular basis for substrate specificity of protein-tyrosine phosphatase 1B, J . Biol. Chem. 273, 26368-26374.

Skolnick, J ., Kihara D. (2001) Defrosting the Froxen Approximation: PROSPECTOR-A new approach to threading, Proteins 42, 319-331.

Skolnick, J ., Kolinski, A., and Ortiz, A. R. (1997) MONSSTER: A method for folding globular proteins with a small number of distance restraints, J. Mol. Biol. 265, 217-241.

Smith, T. F., Gaitatzes, C., Saxena, K., and Neer, E. J . (1999) The WD repeat: A common architecture for diverse functions, Trends Biochem. Sci. 24, 181-185.

Sonnhammer, E. L., Eddy, S. R., Birney, E., Bateman, A., and Durbin, R. (1998) Pfam: Multiple sequence alignments and HMM-profiles of protein domains, Nucleic Acids Res. 26, 320322.

Thomas, J ., Milward, D., Ouzounis, C., Pulman, S., and Carroll, 
M. (2000) Automatic Extraction of Protein Interactions from Scientific Abstracts, Pac. Symp. Biocomput., pp. 541-552.

Tomic, S., Nilsson, L., and Wade, R. C. (2000) Nuclear receptorDNA binding specificity: A COMBINE and Free-Wilson QSAR analysis, J. Med. Chem. 43, 1780-1792.

Tramontano, A. (1998) Homology modeling with low sequence identity, Methods 14, 293-300.

Tsurusaki, Y., Misawa, H., and Yamaguchi, M. (2000) Translocation of regucalcin to rat liver nucleus: I nvolvement of nuclear protein kinase and protein phosphatase regulation [I n Process Citation], Int. J. Mol. Med. 6, 655-660.

Wallace, A. C., Borkakoti, N., and Thornton, J . M. (1997) TESS: A geometric hashing algorithm for deriving 3D coordinate templates for searching structural databases. Application to enzyme active sites, Protein Sci. 6, 2308-2323.

Weichsel, A., Gasdaska, J. R., Powis, G., and Montfort, W. R. (1996) Crystal structures of reduced, oxidized, and mutated human thioredoxins: Evidence for a regulatory homodimer, Structure 4, 735-751.

Xia, T. H., Bushweller, J . H., Sodano, P., Billeter, M., Bjornberg,
O., Holmgren, A., and Wuthrich, K. (1992) NMR structure of oxidized Escherichia coli glutaredoxin: Comparison with reduced E. coli glutaredoxin and functionally related proteins, Protein Sci. 1, 310-321.

Xu, H., Aurora, R., Rose, G. D., and White, R. H. (1999) Identifying two ancient enzymes in Archaea using predicted secondary structure alignment, Nat. Struct. Biol. 6, 750-754.

Yuvaniyama, J ., Denu, J . M., Dixon, J . E., and Saper, M. A. (1996) Crystal structure of the dual specificity protein phosphatase VHR, Science 272, 1328-1331.

Zhang, L., Godzik, A., Skolnick, J., and Fetrow, J. S. (1998) Functional analysis of the Escherichia coli genome for members of the alpha/beta hydrolase family, Fold. Des. 3, 535-548.

Zhang, M., Stauffacher, C. V., Lin, D., and Van Etten, R. L. (1998) Crystal structure of a human low molecular weight phosphotyrosyl phosphatase. Implications for substrate specificity, J . Biol. Chem. 273, 21714-21720.

Zhang, Z., Schaffer, A. A., Miller, W., Madden, T. L., Lipman, D. J ., Koonin, E. V., and Altschul, S. F. (1998) Protein sequence similarity searches using patterns as seeds, Nucleic Acids Res. 26, 3986-3990. 